\title{
An Incentive Compatible Planning Procedure for Public Good Production
}

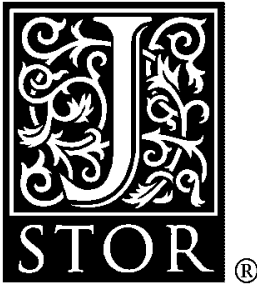

Jerry Green; Jean-Jacques Laffont

The Scandinavian Journal of Economics, Vol. 80, No. 1. (1978), pp. 20-33.

Stable URL:

http://links.jstor.org/sici?sici=0347-0520\%281978\%2980\%3A1\%3C20\%3AAICPPF\%3E2.0.CO\%3B2-5

The Scandinavian Journal of Economics is currently published by The Scandinavian Journal of Economics.

Your use of the JSTOR archive indicates your acceptance of JSTOR's Terms and Conditions of Use, available at

http://www.jstor.org/about/terms.html. JSTOR's Terms and Conditions of Use provides, in part, that unless you have obtained prior permission, you may not download an entire issue of a journal or multiple copies of articles, and you may use content in the JSTOR archive only for your personal, non-commercial use.

Please contact the publisher regarding any further use of this work. Publisher contact information may be obtained at http://www.jstor.org/journals/sje.html.

Each copy of any part of a JSTOR transmission must contain the same copyright notice that appears on the screen or printed page of such transmission.

JSTOR is an independent not-for-profit organization dedicated to and preserving a digital archive of scholarly journals. For more information regarding JSTOR, please contact support@jstor.org. 


\title{
AN INCENTIVE COMPATIBLE PLANNING PROCEDURE FOR PUBLIC GOOD PRODUCTION
}

\author{
Jerry Green \\ Harvard University, Cambridge, Massachusetts, USA
}

Jean-Jacques Laffont

Ecole Polytechnique, C.N.R.S., Paris, France

\begin{abstract}
It is only recently that economic theorists have faced the fact that the proposed allocation mechanisms in economies with public goods might have bad incentive properties. In this paper we introduce a new planning procedure such that truthful revelation of the marginal rates of substitution between public goods and the private good is a dominant strategy at each instant along the procedure, i.e. we obtain much stronger incentive properties than in the planning procedures proposed in the literature. We demonstrate the convergence of this new planning procedure for economies with a single public good and explore its properties-finding mixed results-in more general systems.
\end{abstract}

\section{Introduction}

It is only recently that economists have faced the fact that the proposed allocation mechanisms in economies with public goods might have bad incentive properties. Malinvaud (1971) presented three dynamic planning procedures for such economies, in each of which there exists the incentive for incorrect revelation of preferences. In section 6 of his paper, Malinvaud comments on this problem and pursues one of his mechanisms under the assumption that a Nash equilibrium in professed preferences is found at each date. See also Roberts (1976). In this paper we introduce a new planning procedure with incentive properties stronger than those possessed by the Malinvaud mechanism. We demonstrate its convergence for economies with a single public good, and explore its properties-finding mixed results-in more general systems.

Recent research on the incentives problem has proceeded along two principle lines. Clarke (1971), Hurwicz (1972), Groves (1973) Groves and Ledyard (1977) have used a global criterion. They have endowed agents with messages aimed at effectively transmitting the relevant aspects of their whole utility 
functions. In a different spirit Drèze and de la Vallée Poussin (1971) and Malinvaud (1971) have constructed a continuous time procedure for which the messages of agents at each instant are their marginal rates of substitution, that is, local information about their preferences is transmitted. ${ }^{1}$

These two approaches have developed without much interaction and with somewhat contrasting results as noted by Roberts (1976). The main purpose of this paper is to show the analogous role played by the assumption of separability of utility functions in global approaches on the one hand and the assumption of myopia in local dynamic approaches on the other.

We construct a dynamic version of the Clarke-Groves mechanism which, in a static model, "essentially" yields a Pareto optimal allocation with revelation of the truth as a dominant strategy when utility functions are separable. We show that in the dynamic version the same results obtain for non separable utility functions when we postulate a myopic form of behavior. The symmetric role of separability and myopia is easily understood, when one realizes that at each instant, the welfare of an agent, represented by the time derivative of the utility function is linear in the time derivative of the quantity of public good and the time derivative of the quantity of private good.

Another attractive feature of the continuous time dynamic procedures in contrast with global methods concerns informational requirements. First, the agent has only to reveal his preferences along the actual path followed by the process and not for the whole range of the variables. Second, the level of accuracy that can be obtained from agents willing to transmit their preferences depends on the way they are elicited and to ask local questions along a path seems to be a good way to proceed. Moreover the maximization problem of the center is much simpler at each instant-though of course it must be done infinitely quickly.

In Section II, we describe our mechanism with a single public good. Generalizations to several public goods are provided in Section III.

\section{A Procedure with One Private Good and One Public Good}

We consider an economy with $N$ consumers and one private good, denoted $y$, and one public good, denoted $x$. Let $u^{i}\left(x, y^{i}\right)$ be the utility function of the $i$ 'th consumer as a function of his consumption of public good and private good respectively; $u^{i}(.,$.$) is assumed to be strictly concave and twice contin-$ uously differentiable with:

1 Roberts (1976) and Henry (1976) have shown that under myopic behavior if a Nash equilibrium in announced strategies is obtained at each instant, truthful revelation is not obtained but a Pareto optimum is reached. This interesting result is difficult to interpret because a discrete formulation of the Nash game at each instant $t$ shows that the Nash equilibrium is unstable. In a discrete formulation of MDP, Henry (1976) shows the need for an assumption like "when agents are indifferent, they say the truth" to obtain stability. 
$u_{x}^{i}\left(x, y^{i}\right)=\frac{\partial u^{i}}{\partial x}\left(x, y^{i}\right) \geqslant 0$

$u_{y}^{i}\left(x, y^{i}\right)=\frac{\partial u^{i}}{\partial y}\left(x, y^{i}\right)>0$

The marginal rate of substitution between public good and private good is denoted:

$\pi^{i}\left(x, y^{i}\right)=\frac{u_{x}^{i}\left(x, y^{i}\right)}{u_{y}^{i}\left(x, y^{i}\right)}$

We assume that the utility function is defined for any value $y^{i} \in R$, so that we do not have to worry about bankruptcy (see, however, the discussion below for different ways of treating bankruptcy).

Finally, consumers have endowments $\left(w^{i}\right)$ in the private good.

The production possibilities are described by a twice differentiable increasing function $G(x)$ which specifies the amount of private good necessary to produce any given amount $x$ of the public good.

The marginal cost of producing an additional unit of public good is denoted $\gamma(x)$ with

$\gamma(x)>0 \quad$ and $\quad \gamma^{\prime}(x)>0 \quad$ if $x>0, \quad \gamma(0)=0, \quad \gamma^{\prime}(0)=0$.

Lemma 1. An allocation $z=\left(x, y^{1}, \ldots, y^{N}\right)$ is a Pareto optimum iff

$\sum_{i=1}^{N} \pi^{i}\left(x, y^{i}\right)=\gamma(x)$

$G(x)+\sum_{i=1}^{N} y^{i}=\sum_{i=1}^{N} w^{i}$

Proof. Obvious.

We consider a (time) continuous procedure starting at date 0 , from the allocation $\left(0, w^{1}, \ldots, w^{N}\right)$. At each instant $t$, each agent is asked to report his marginal rate of substitution between the public good and the private good. Let $\psi^{i}(t)$ be his stated marginal rate of substitution at date $t$. There is no $\grave{a}$ priori restriction on the functions $\psi^{i}(),. i=1, \ldots, N$.

Let $\delta^{i}$ be the cost share imputed to agent $i$ with $\sum_{i=1}^{N} \delta^{i}=1$.

At each date $t$, we define the set of pivotal individuals $P(t)$ as follows:

$i \in P(t) \Leftrightarrow\left(\sum_{i} \psi^{i}(t)-\gamma(x(t))\right)\left(\sum_{j \neq i}\left(\psi^{j}(t)-\delta^{j} \gamma(x(t))\right)\right)<0$.

An agent is pivotal if his statement leads to a. different sign for the aggregate net willingness to pay.

The procedure is then defined by the following system of differential equations: 


$$
\begin{aligned}
\dot{x}(t) & =+1 \quad \text { if } \quad \sum_{i} \psi^{i}(t)-\gamma(x(t))>0 \\
& =0 \quad \text { if } \quad \sum_{i} \psi^{i}(t)-\gamma(x(t))=0 \\
& =-1 \quad \text { if } \quad \sum_{i} \psi^{i}(t)-\gamma(x(t))<0 . \\
\dot{S}(t) & =-\sum_{i \in P(t)} \sum_{j \neq i}\left[\psi^{j}(t)-\delta^{j} \gamma(x(t))\right] \dot{x}(t) \\
\dot{y}^{i}(t) & =-\delta^{i} \gamma(x(t)) \dot{x}(t)+\sum_{j \neq i}\left[\psi^{j}(t)-\delta^{j} \gamma(x(t))\right] \dot{x}(t)+\frac{1}{N} \dot{S}(t) \quad \text { if } \quad i \in P(t) \\
& =-\delta^{i} \gamma(x(t)) \dot{x}(t)+\frac{1}{N} \dot{S}(t) \quad \text { if } \quad i \notin P(t) .
\end{aligned}
$$

Equation (2.3) specifies the adjustment speed of the quantity of public good, according to the sign of the aggregate net willingness to pay.

Equations (2.5) and (2.6) describe the change in the endowment of private good for pivotal and non pivotal agents. Consider first the mechanism without the surplus $\dot{S}(t)$. For non-pivotal agents the change in private good is $-\delta^{i} \gamma(x(t)) \dot{x}(t)$, i.e. the imputed cost of the additional unit of public good. In addition, a pivotal agent has to pay $\sum_{j \neq i}\left[\psi^{j}(t)-\delta^{j} \gamma(x(t))\right] \dot{x}(t)$, i.e. the sum of net willingnesses to pay of the other agents. If the procedure was run this way it would generate a surplus at each date since

$\gamma \dot{x}(t)+\sum_{i=1}^{N} \dot{y}^{i}(t)=\sum_{i \in P(t)} \sum_{j \neq i}\left[\psi^{j}(t)-\delta^{j} \gamma(x(t))\right] \dot{x}(t)<0$

instead of

$\gamma \dot{x}(t)+\sum_{i=1}^{N} \dot{y}^{i}(t)=0$

from (2.2).

In order to close the system we must redistribute this surplus. This is done by giving back $(1 / N) \hat{S}(t)$ equally to every agent. ${ }^{1}$

Now, we have to make behavioral assumptions to explain how agents determine their answers. We will consider two possibilities based on our previous work on the static model, see Green \& Laffont (1976).

Assumption 1. Each agent has a myopic behavior, i.e. defines his optimal answer at date $t$ by considering only the increase of utility he can obtain at date $t$, namely

$\dot{u}^{i}=\frac{\partial u^{i}}{\partial x} \dot{x}+\frac{\partial u^{i}}{\partial y^{i}} \dot{y}^{i}$

\footnotetext{
1 More general ways of distributing the surplus could of course be assumed, as long it is distributed among a large enough group.
} 
This is, of course, the critical assumption which allows us to bypass the separability assumption, by considering at each instant the linearized approximation of the utility function which is indeed separable.

Assumption 2. For large $N$, each agent neglects the impact of his answer on $(1 / N) \dot{S}(t)$, in determining his optimal answer.

The procedure with A 2 is referred to as procedure A. ${ }^{1}$ The justification for assumption 2 is that if agents think that the marginal rates of substitution of the others are an independent random sample from some population, then the empirical distribution of $\dot{S} / \sqrt{N}$ would converge to a distribution with finite mean and variance. Therefore $\dot{S} / N$ would be statistically negligible. See Green \& Laffont (1978, Chapter 9). Of course the fact that $S / \sqrt{N}$ is small does not by itself mean that its response to changes in $\psi_{i}(t)$ would be negligible. However, we have shown in Green et al. (1976) that as $N$ becomes large, the optimal strategy of each agent converges to the truth. Assumption 2, therefore, is justified as an approximation in this sense.

Let us denote $\dot{u}^{i}\left(\psi^{i}(t), \psi^{-i}(t)\right)$ the anticipated change in utility for agent $i$, if he says $\psi^{i}(t)$ when the other agents answer $\psi^{-i}(t)=\mid \psi^{\mathbf{1}}(t), \ldots, \psi^{i-1}(t)$, $\psi^{i+1}(t), \ldots, \psi^{N}(t) \mid$.

Lemma 2. Under A1-A2, revelation of the truth at each instant $t$, is a dominant strategy.

Proof. We want to show that

$\Delta^{i}=\dot{u}^{i}\left(\pi^{i}(t), \psi^{-i}(t)\right)-\dot{u}^{i}\left(\psi^{i}(t), \psi^{-i}(t)\right) \geqslant 0$

for any $\psi^{i}(t), \psi^{-i}(t)$.

Below, we check that it is true for several cases leaving symmetric situations to the reader.

If

$\sum_{j \neq i} \psi^{j}(t)+\pi^{i}(t)>\gamma(t)$

$\sum_{j \neq i} \psi^{j}(t)+\psi^{i}(t)>\gamma(t)$

and,

$\sum_{j \neq i}\left(\psi^{j}(t)-\delta^{j} \gamma(t)\right)<0$

then

$$
\begin{aligned}
\Delta^{i}=u_{y}^{i}\left(x(t), y^{i}(t)\right)\left[\pi^{i}(t)\right. & -\delta^{i} \gamma(t)+\sum_{j \neq i}\left(\psi^{j}(t)-\delta^{j} \gamma(t)\right)+\frac{1}{N} \dot{S}(t) \\
& \left.-\pi^{i}(t)+\delta^{i} \gamma(t)-\sum_{j \neq i}\left(\psi^{j}(t)-\delta^{j} \gamma(t)\right)-\frac{1}{N} \dot{S}(t)\right]=0 .
\end{aligned}
$$

\footnotetext{
1 The transfer function without the rebate used at each instant is not exactly a ClarkeGroves mechanism; the exact transposition of the Clarke mechanism would give larger surpluses and require at the equilibrium additional payments which might destroy stability with non separable utility functions (at least in a discrete version of the procedure).
} 
If

$\sum_{j \neq i} \psi^{j}(t)+\pi^{i}(t)>\gamma(t)$

$\sum_{j \neq i} \psi^{j}(t)+\psi^{i}(t)=\gamma(t)$

and,

$\sum_{j \neq i}\left[\psi^{j}(t)-\delta^{j} \gamma(t)\right]<0$

then

$\Delta^{i}=u_{y}^{i}\left(x(t), y^{i}(t)\right)\left[\sum_{j \neq i} \psi^{j}(t)+\pi^{i}(t)-\gamma(t)+\frac{1}{N} \dot{S}(t)\right] \geqslant 0$.

If

$\sum_{j \neq i} \psi^{j}(t)+\pi^{i}(t)=\gamma(t)$

$\sum_{j \neq i} \psi^{j}(t)+\psi^{i}(t)>\gamma(t)$

and

$\sum_{j \neq i}\left[\psi^{j}(t)-\delta^{j} \gamma(t)\right]>0$

we know that

$\pi^{i}(t)<\delta^{i} \gamma(t)$,

and hence,

$\Delta^{i}=u_{y}^{i}\left(x(t), y^{i}(t)\right)\left[-\pi^{i}(t)+\delta^{i} \gamma(t)\right]>0$.

If

$\sum_{j \neq i} \psi^{j}(t)+\pi^{i}(t)=\gamma(t)$

$\sum_{j \neq i} \psi^{j}(t)+\psi^{i}(t)<\gamma(t)$

and,

$\sum_{j \neq i}\left(\psi^{i}(t)-\delta^{j} \gamma(t)\right)>0$

then

$\Delta^{i}=u_{y}^{i}\left(x(t), y^{i}(t)\right)\left[\pi^{i}(t)-\delta^{i} \gamma(t)+\sum_{j \neq i}\left(\psi^{j}(t)-\delta^{j} \gamma(t)\right)\right]=0$.

Q.E.D.

This lemma is not covered in Green \& Laffont (1976), but is closely related. In that paper, we break a tied situation in favor of one of the two projects arbitrarily. To reach an equilibrium in the dynamic procedure, we must introduce a third project when $\sum_{i} \pi^{i}=\gamma$, namely, keeping the status quo and 
neither increasing nor decreasing the level of the public good. Ties are now to be broken in favor of this decision. However, there are here further constraints on the utility functions (linearity in public good) which with A2 still make truthful revelation a dominant strategy.

Lemma 3. A stationary point of the procedure is a Pareto optimum.

Proof. Obvious.

Assumption 3. There exists a level of public good production $\bar{x}$ such that for any $\left(y^{1}, \ldots, y^{N}\right), \sum_{i} \pi^{i}\left(\bar{x}, y^{i}\right)-\gamma(\bar{x})$ is negative.

Theorem 1. The procedure A converges to a Pareto optimum.

Proof. At date zero $\dot{x}(0)>0$ from our assumptions.

At each instant, condition (2.2) of Pareto optimality is satisfied because the procedure is closed; $\dot{x}(t)$ cannot become negative for $t>0$ because since $\sum_{i} \pi_{i}-\gamma$ is continuous by the intermediate value theorem it would have to take the zero value where the procedure would stop.

Therefore $x(t)$ is increasing and bounded by $\bar{x}$ (see assumption 3 ). Therefore, it converges to $x^{*}>0$. Let $\left(y^{1 *}, \ldots, y^{N *}\right)$ be the associated distribution of the private good.

From (2.3) $\dot{x}^{*}=0$, implies $\sum_{i=1}^{N} \pi^{i}\left(x^{*}, y^{i *}\right)=\gamma\left(x^{*}\right)$, and $\dot{y}^{i *}=0, i=1, \ldots, N$ hence the asymptotic Pareto optimality.

Q.E.D.

The procedure converges in a finite amount of time $T^{*}$ defined as

$$
\int_{0}^{T^{*}} \dot{x}(t) d t=x^{*}, \text { or } T^{*}=x^{*}
$$

The existence of solutions to the system of differential equations is no problem, since the right hand side equations are continuously differentiable, with the exception of (2.3) which does not matter because $\dot{x}$ never changes sign. The continuity of the right-hand side of (2.4), (2.5), (2.6) in spite of the introduction of pivotal agents is due to the fact that when an agent becomes a pivot or ceases to be a pivot the quantity $\sum_{j \neq i}\left(\psi^{j}(t)-\delta^{j} \gamma(t)\right)$ takes the value zero.

It is important to observe that this procedure is not individually rational, in the sense of improving the welfare of everybody with respect to the initial situation, unless very strong assumptions are made (such as "the public good is necessary") which de facto make the initial position the worst for every agent. It is easy to see in procedure $A$ that the utility of an agent increases whenever he is pivotal since: 


$$
\begin{aligned}
\dot{u}^{i} & =u_{y}^{i}\left(\pi^{i} \dot{x}-\delta^{i} \gamma \dot{x}+\sum_{j \neq i}\left(\pi^{j}-\delta^{j} \gamma\right) \dot{x}+\frac{1}{N} \dot{S}\right) \\
& =u_{y}^{i}\left(\left(\sum_{i} \pi^{i}-\gamma\right) \dot{x}+\frac{1}{N} \dot{S}\right)>0 .
\end{aligned}
$$

However, when he ceases to be pivotal his utility may well decrease, if his net willingness to pay $\left(\pi^{i}-\delta^{i} \gamma\right)$ is negative and if the rebate he gets $(1 / N) \dot{S}$ does not compensate him fully.

In an economy where the initial situation corresponding to a distribution of the private good has no reason to be fair, the requirement of individual rationality looses much of its appeal. It is clear that the spirit of the procedures proposed here is to go beyond the Pareto optimal criterion by using a cardinal representation of the utility functions and by maximizing the social welfare represented by the sum of utilities. By choosing equal shares of the cost, the procedure can be made equitable in the following sense: if the agents consider the procedure before knowing their own preferences, in the spirit of the Rawlsian approach, no particular agent is favored. However, this procedure is certainly not optimal for extremely risk averse agents, as required by Rawls.

The lack of individual rationality creates also the possibility of bankruptcy. If one may consider that we are dealing with small projects relatively to the wealth of the agents, then, there is no real problem of bankruptcy. But if it is desired to handle big public projects with such a method, bankruptcy must be faced. An agent may be bankrupted for two reasons. On the one hand he may be unable to pay his ex ante imputed share of the project; in that case one may legitimely assume that the Center knows the endowments in sufficient detail so that he does not impute shares which could bankrupt some agents. On the other hand, he may have to make pivotal payments beyond his means. Note, however, that the mechanism is such that a pivotal payment is always less than the net marginal willingness to pay. Therefore, if one bounds the marginal rate of substitution, it is possible to limit the eventual pivotal payments to an amount below the endowment net of the cost share.

The procedure described above may be slightly modified to allow different interpretations. Instead of redistributing the surplus one may choose to destroy it in order to be sure of not distorting incentives. The loss in efficiency can then be measured in the private good as:

$L=\int_{0}^{T^{*}} \dot{S}(t) d t=\int_{0}^{T^{*}} \sum_{i \in P(t)}\left[\sum_{j \neq i} \psi^{j}(t)-\delta^{j} \lambda(t)\right] d t$

where $T^{*}$ is the convergence time of the procedure.

To bound the per capita efficiency loss, and to show that it tends to be small in large systems, we consider an economy with $r \cdot n$ agents defined as 
follows. There are $n$ agents described as above, and the economy consists of an $r$-fold replica of these agents. Let us assume that net willingnesses to pay always lie in the interval $[-A,+A]$.

$A$ bound on $L$ is constructed by considering the first date, $T_{1}(r)$, at which pivotal payments by any agent are possible (that is, the first date at which $\sum_{i} \psi^{i}-\gamma=A$ ). When pivotal payments are being made, the rate of payments at any instant cannot be greater than $r n A / 2$. Therefore $r n A / 2 \cdot\left(T^{*}-T_{1}(r)\right)$ is an upper bound for $L$.

We approximate $T_{1}(r)$ by writing the Taylor series for

$$
Z(t)=\sum_{i=1}^{r n} \psi^{i}-\gamma
$$

around $T^{*}$, where it is zero by definition. This is

$Z(t)=\left(t-T^{*}\right) \dot{Z}\left(T^{*}\right)+\frac{\left(t-T^{*}\right)^{2}}{2} \ddot{Z}(\tilde{t}) \quad$ for $\tilde{t} \in\left(t, T^{*}\right)$.

Since $\dot{Z}\left(T^{*}\right)=0$ and $\ddot{Z}(\tilde{t})=r \sum_{i=1}^{n} \ddot{v}(\tilde{t})$ we have

$$
T^{*}-T_{1}(r)=\frac{\sqrt{A}}{\left(r \sum_{i=1}^{u} \ddot{v}_{i}(\tilde{t})\right)^{\frac{1}{2}}}
$$

Thus

$$
L<\frac{r^{\frac{1}{2}} A^{\frac{3}{2}} n}{2\left(\sum_{i=1}^{n} \ddot{v}_{i}(t)\right)^{\frac{1}{2}}} .
$$

By bounding $\ddot{v}_{i}(\tilde{t})$ below we have a bound on $L$ such that the per capita efficiency loss, $L / r n$, is decreasing with $r$.

The bound on $\ddot{v}_{i}(\tilde{t})$ depends on both the dynamics of the process and on the underlying utility functions. If these utility functions are separable, so that $\psi$ depends only on $y$, then $\ddot{v}_{i}(\tilde{t})$ will be bounded if there is a lower bound on $\psi^{\prime}$. More generally, the bound on $\ddot{v}_{i}(\tilde{t})$ cannot be derived without further restrictions. For example, in the case of homothetic utility functions, assumptions on the elasticity of substitution and on the curvature of the cost function would suffice.

Therefore, we have developed a continuous time procedure which at the cost of a somewhat bounded rationality or a slight inefficiency gives satisfactory answers both in terms of incentives and efficiency.

\section{Several Public Goods}

The generalization of the above results to the case of several public goods faces the difficulty of proving stability. In a first subsection we construct a 
procedure converging to a Pareto optimum when the utility functions are separable. ${ }^{1}$ In subsection $\mathbf{B}$, we discuss the difficulties of proving stability without such an assumption.

\section{A. Separability}

We assume here that the utility functions of the $N$ agents can be written: $u^{i}\left(x, y^{i}\right)=y^{i}+v^{i}\left(x_{1}, \ldots, x_{K}\right)$

where $K$ is the number of public goods and $x=\left(x_{1}, \ldots, x_{R}\right)$. The other assumptions and notations of Section II are trivially generalized here.

A Pareto optimum allocation $z=\left(x_{1}, \ldots, x_{K}, y^{1}, \ldots, y^{N}\right)$ is now characterized by:

$\sum_{i=1}^{N} \pi_{k}^{i}\left(x, y^{i}\right)=\gamma_{k}(x), \quad k=1, \ldots, K$

$G(x)+\sum_{i=1}^{N} y^{i}=\sum_{i=1}^{N} w^{i}$

We define imputed 'cost shares for each public project $\delta_{k}^{i}, i=1, \ldots, N$; $k=1, \ldots, K$, as well as a set of pivotal individuals $P_{k}(t)$ for each public project.

$i \in P_{k}(t) \Leftrightarrow\left(\sum \psi_{k}^{i}(t)-\gamma_{k}(x(t))\right)\left(\sum_{j \neq i} \psi_{k}^{j}(t)-\delta_{k}^{j} \gamma_{k}(x(t))\right)<0$.

The procedure is then defined by:

$$
\begin{aligned}
\dot{x}_{k}(t)=+1 \quad \text { if } \quad \sum_{i} \psi_{k}^{i}(t)-\gamma_{k}(x(t))>0 \\
=0 \quad \text { if } \quad \sum_{i} \psi_{k}^{i}(t)-\gamma_{k}(x(t))=0 \\
=-1 \quad \text { if } \quad \sum_{i} \psi_{k}^{i}(t)-\gamma_{k}(x(t))<0 \quad k=1, \ldots, K .
\end{aligned}
$$

$\dot{S}_{k}(t)=-\sum_{i \in P_{k}(t)}\left[\sum_{j \neq i} \psi_{k}^{j}(t)-\delta_{k}^{j} \gamma_{k}(x(t))\right] \dot{x}_{k}(t) \quad k=1, \ldots, K$

$\dot{y}^{i}(t)=-\sum_{k} \delta_{k}^{i} \gamma_{k}(x(t)) \dot{x}_{k}(t)+\sum_{k} d_{k}^{i} \sum_{j \neq i}\left[\psi^{j}(t)-\delta_{k}^{j} \gamma_{k}(x(t))\right]+\frac{1}{N} \sum_{k} \dot{S}_{k}(t)$

with

$d_{k}^{i}=0 \quad$ if $\quad i \notin P_{k}(t)$

$d_{k}^{i}=1 \quad i \in P_{k}(t)$.

It is immediate to show that under the analogue of $\mathrm{A} 1-\mathrm{A} 2$ ( $\mathrm{A} 1^{\prime}, \mathrm{A} 2^{\prime}$ ) truthful revelation of preferences is a dominant strategy for each agent.

1 Note however that the informational requirements are weaker in this procedure than if we used directly a Groves mechanism which elicits the whole functions $v^{i}($.$) .$ 
Assumption $3^{\prime}$. For any $k$, there exists a level of public good production $\bar{x}_{k}$ such that for any $x_{-k}=\left(x_{1}, \ldots, x_{k-1}, x_{k+1}, \ldots, x_{K}\right)$ and any $y^{1}, \ldots, y^{N}$ $\sum_{i} \pi_{k}^{i}\left(\bar{x}_{k}, x_{-k}, y^{i}\right)-\gamma_{k}\left(\bar{x}_{k}, x_{-k}\right)$

is negative.

The system of differential equations so described is a complex one, in particular because of a number of discontinuities in the right hand side. First, the speed of adjustment for the different public goods is discontinuous. In addition, when the speed of adjustment for a given public good changes sign at instant $t_{0}$ the set of pivotal agents changes, and eventhough the sum of pivotal payments is the same when

$t \rightarrow t_{0}$
$t>t_{0}$ or when $\begin{aligned} & t \rightarrow t_{0}, \quad \text { at } t=t_{0} \\ & t<t_{0}\end{aligned}$

this sum is zero at $t_{0}$ because $k=0$. Also, the rate of monetary transfer to an agent who at $k=0$ ceases to be (or becomes) pivotal is discontinuous. Note however that when the speed of adjustment is unchanged, the transfer an agent who ceases to be (or becomes) pivotal is unchanged. Therefore, the system of differential equations has only discontinuities at instants when the quantities of some (and not all) public goods are stationary. ${ }^{1}$

Assuming the existence of a solution to (3.4), (3.5) and (3.6) we can then prove.

Theorem 2. Under $A 1^{\prime}, A 2^{\prime}, A 3^{\prime}$, the procedure $A$ with many public goods converges to a Pareto optimum if preferences are "separable".

Proof. Clearly a stationary point of the procedure is a Pareto optimum. Consider the following Lyapunov function:

1 It may be hoped that the methods of Champsaur et al. (1977) can be used to establish the existence of solutions to (3.4), (3.5) and (3.6). To do so one would consider an analogue system of differential equations in which the right hand side is set valued and contains the value given by (3.4), (3.5) and (3.6) at every point. Specifically, one chooses these sets so as to form the smallest convex-valued, upper hemi-continuous correspondence containing the required discontinuous function. For example, (3.4) would be modified to read

$$
\begin{aligned}
& \dot{x}_{k}(t)=\quad+1 \quad \text { if } \quad \sum_{i} \psi_{k}^{i}(t)-\gamma_{k}(x(t))>0 \\
& =[-1,+1] \quad \text { if } \quad \sum_{i} \psi_{k}^{i}(t)-\gamma_{k}(x(t))=0 \\
& =\quad-1 \text { if } \sum_{i} \psi_{k}^{i}(t)-\gamma_{k}(x(t))<0 .
\end{aligned}
$$

A solution to such a system is a function whose derivatives are members of the indicated sets at every point in time. Solutions to this convexified system are known to exist, and their properties are developed in Castaing \& Valadier (1969). The method of Champsaur et al. (1977) is to show that such solutions also satisfy the original system. At present this is an open question. Analogous extensions of discontinuous processes in economics have been made by Henry (1972) and Hori (1975). 
$V(t)=\sum_{i} u^{i}\left(x, y^{i}\right)$

Clearly,

$V(t)>0 \quad$ and $\quad \dot{V}(t)=\sum_{i}\left[\ddot{y}^{i}+\sum_{k} \pi_{k}^{i} \dot{x}_{k}\right]$

Using (3.6) we have

$\dot{V}(t)=\sum_{i} \sum_{k}\left[\pi_{k}^{i}-\delta_{k}^{i} \gamma_{k}(x(t))\right] \dot{x}_{k}>0$

and $\dot{V}(t)=0$ iff the allocation is Pareto optimum.

From $\mathrm{A} 3{ }^{\prime}$, with (3.7), $x_{k}$ must be bounded above for every $k$. Therefore $V(t)$ must be bounded above.

$V(t)$ is an increasing function of time which is bounded above. Given that solutions exist, theorem 6.2 in Champsaur, Drèze and Henry (1977), implies that the procedure is quasi stable - that is, any limit point of any trajectory is a stationary point. It is immediate then to obtain stability by using the strict quasiconcavity of the utility functions and the fact that any stationary point is a Pareto optimum.

Q.E.D.

\section{B. Instability without Separability}

Without separability the transfers in the private good may disturb the stability of the procedure. Suppose there are two public goods. With appropriate concavity assumptions, it is clear that the change in the production of public goods brings us closer to the Pareto surface as $\left|\sum_{i} \pi_{k}^{i}-\gamma_{k}\right|, k=1,2$ decrease. We approach a Pareto allocation with given levels of public good productions. But, in the procedure, we are obliged to make transfers in the private good to an agent who will favor a different structure of public goods. But, as we approach this different mixture, transfers are now made to another agent who reverses the trajectory towards the previous combination. During a cycle, the utility level of one agent increases and then decreases while for another agent it is the opposite. This is made possible by the lack of individual rationality of the procedure. This phenomenon cannot occur with separability since the transfers in private good do not affect the decisions about public goods.

Hereafter we give the elements of an example with piecewise linear utility functions where instability occurs.

The example involves two public goods with constant marginal costs equal to 2 and two agents who share equally the costs of the public goods: $\delta^{1}=\delta^{2}=\frac{1}{2}$. Let $M^{i}$ be the initial amount of private good hold by each agent, $i=1,2 . M^{i}$ is large enough so that no bankruptcy occurs along the trajectories.

The preferences are locally defined along the trajectory as follows; appropriate increasing transformations can be used to make these preferences continuous. 


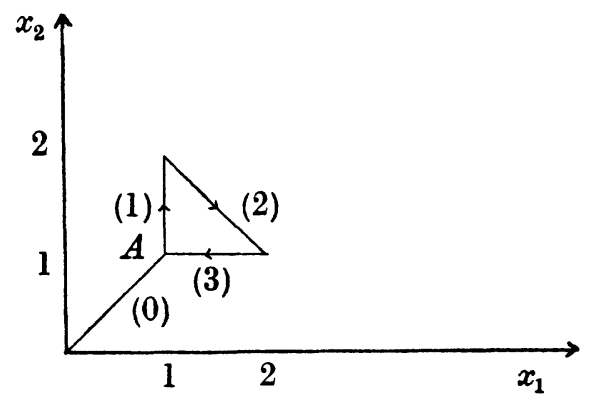

Fig. 1

\section{Agent 1}

For $M^{1}-2 \leqslant y^{1} \leqslant M^{1}$

$$
0 \leqslant x_{1}=x_{2}<1 \quad \text { then } U^{1}=2 x_{1}+2 x_{2}+y^{1}
$$

For $M^{1}-2.75 \leqslant y^{1} \leqslant M^{1}-2$

$$
\begin{aligned}
x_{1} & =1 \\
1 \leqslant x_{2} & <2 \quad \text { then } U^{1}=x_{1}+\frac{1}{2} x_{2}+y^{1}
\end{aligned}
$$

For $\quad M^{1}-3.25 \leqslant y^{1} \leqslant M^{1}-2.75$

$$
\begin{gathered}
x_{1}+x_{2}=3 \\
1 \leqslant x_{1}<2,1<x_{2} \leqslant 2 \text { then } U^{1}=3 x_{1}+y^{1}
\end{gathered}
$$

For $M^{1}-3.25 \leqslant y^{1} \leqslant M^{1}-2$

$$
\begin{aligned}
x_{2} & =1 \\
1 \leqslant x_{1} & \leqslant 2 \quad \text { then } U^{1}=1.5 x_{1}+x_{2}+y^{1}
\end{aligned}
$$

Agent 2

For $M^{2}-2 \leqslant y^{2} \leqslant M^{2}$

$$
0 \leqslant x^{1}=x^{2}<1 \quad \text { then } U^{2}=2 x_{1}+2 x_{2}+y^{2}
$$

For $M^{2}-3.25 \leqslant y^{2} \leqslant M^{2}-2$

$$
\begin{aligned}
x_{1} & =1 \\
1 \leqslant x_{2} & <2 \quad \text { then } U^{2}=x_{1}+4 x_{2}+y^{2}
\end{aligned}
$$

For $M^{2}-3.25 \leqslant y^{2} \leqslant M^{2}-2.75$

$$
\begin{gathered}
x_{1}+x_{2}=3 \\
1 \leqslant x_{1}<2,1 \leqslant x_{2} \leqslant 2 \text { then } U^{2}=0.5 x_{1}+1.5 x_{2}+y^{2}
\end{gathered}
$$

For $M^{2}-3.25 \leqslant y^{2} \leqslant M^{2}-2$

$$
\begin{aligned}
x_{2} & =1 \\
1 \leqslant x_{1} & \leqslant 2 \quad \text { then } U^{2}=0.25 x_{1}+x_{2}+y^{2} .
\end{aligned}
$$

In the space of public goods the trajectory is as shown in Fig. 1. 
In the region (0) (see Fig. 1), there is no pivot, and both projects are constructed in increasing quantity. Then we enter region (1) with incomes $M^{1}-2$ and $M^{2}-2$ for the two agents. Then agent 2 is a pivot for the second public good and he has to pay $\frac{1}{2}$ with a rebate of $\frac{1}{4}$, i.e. $\frac{1}{4}$. In region (1) his income decreases from $\mathrm{M}^{2}-2$ to $M^{2}-3.25$ since he has also to pay his share of the cost of the second public good i.e. 1. Agent 1 on the other hand receives $\frac{1}{4}$ and has to pay his share of the cost. In region (2), agent 1 is a pivot for public good, because he causes it to be increased, and a pivot for public good 2 but in the opposite sense. Finally in region (3), agent 2 is a pivot in the downward direction. Then we are back at $A$ and cycle indefinitely in (1), (2), (3).

\section{References}

Bowen, H.: The interpretation of voting in the allocation of economic resources. Quarterly Journal of Economics 58, 27-48, 1943.

Castaing, C. \& Valadier, M.: Equations differentielles multivoques dans les espaces localement convexes. Revue Francaise d'Informatique et de Recherche Opérationnelle 16, 1969.

Champsaur, P., Drèze, J. \& Henry, C.: Dynamic processes in economic theory. Econometrica 45, 273-294, 1977.

Drèze, J. \& de la Vallée Poussin, D.: A tatonnement process for public goods. The Review of Economic Studies 38, 133$150,1971$.

Green, J., Kohlberg, E. \& Laffont, J. J.: Partial equilibrium approach to the free rider problem. Journal of Public Economics 6, 375-394, 1976.

Green, J. \& Laffont, J. J.: Révélation des préférences pour les biens publics. lère Partie. Caractérisation des mécanismes satisfaisants. D.P. Ecole Polytechnique (1976) to appear in Cahiers du Séminaire d'Econométrie.

Green, J. \& Laffont, J. J.: Incentives in public decision making. North-Holland Press, 1978 (forthcoming).

Groves, T.: Incentives in teams. Econometrica 41, 617-631, 1973.

Groves, T. \& Ledyard, J.: Optimal allocation of public goods: a solution to the "free rider" problem. Econometrica 45, 783-810, 1977.

Henry, C.: Differential equations with discontinuous right hand side for planning procedures. Journal of Economic Theory 4, 545-551, 1972.

Hori, H.: The structure of equilibrium points in Heal's process. Review of Economic Studies 43, 457-467, 1975.

Hurwicz, L.: On informationally decentralized systems. In Decision and organization: a volume in honor of Jacob Marschak (ed. C. McGuire and R. Radner). North-Holland, Amsterdam, 1972.

Malinvaud, E.: A planning approach to the public good production. Swedish Journal of Economics 73, 96-112, 1971.

Roberts, J.: Incentives in planning procedures for the provision of public goods. C.O.R.E. D.P. no 7611, 1976. 